\title{
STRONG CONVERGENCE FOR $m$-PAIRWISE NEGATIVELY QUADRANT DEPENDENT RANDOM VARIABLES
}

\author{
Yongfeng Wu And AndRew Rosalsky \\ Tongling University and Soochow University, China and University of \\ Florida, USA
}

\begin{abstract}
Complete convergence and the Marcinkiewicz-Zygmund strong law of large numbers for sequences of $m$-pairwise negatively quadrant dependent ( $m$-PNQD) random variables is studied in this paper. The results obtained extend and improve the corresponding theorems of Choi and Sung ([4]) and $\mathrm{Hu}$ et al. ([9]). A version of the Kolmogorov strong law of large numbers for sequences of $m$-PNQD random variables is also obtained.
\end{abstract}

\section{INTRODUCTION}

The following concept of negatively quadrant dependent random variables was introduced by Lehmann ([12]).

Definition 1.1. Two random variables $X$ and $Y$ are said to be negatively quadrant dependent $(N Q D)$ if

$$
P(X \leq x, Y \leq y) \leq P(X \leq x) P(Y \leq y) \text { for all } x \text { and } y \in \mathbb{R} .
$$

A finite or infinite sequence of random variables is said to be pairwise $N Q D$ (PNQD) if every two random variables in the sequence are NQD. It is well known and easy to show that random variables $X$ and $Y$ are NQD if and only if

$$
P(X>x, Y>y) \leq P(X>x) P(Y>y) \text { for all } x \text { and } y \in \mathbb{R} .
$$

In many stochastic models, an independence assumption among the random variables in the model is not a reasonable assumption since they may be "repelling" in the sense that increases in any of the random variables often

2010 Mathematics Subject Classification. 60F15.

Key words and phrases. $m$-pairwise negatively quadrant dependent random variables, complete convergence, strong law of large numbers. 
correspond to decreases in the others. Thus the assumption of PNQD is often more suitable than the classical assumption of independence.

A major survey article concerning a general "theory of negative dependence" was prepared by Pemantle ([20]). That article discussed the relationship between various definitions of "negative dependence", outlines some possible directions that the theory can take, and provides some interesting conjectures.

We now define a more general dependence structure which contains PNQD as a special case. This dependence structure was introduced by Anh ([2]) for a finite set of random variables. Definition 1.2 is the same as that of Anh ([2]) except that it is for a sequence of random variables rather than for a finite set.

Definition 1.2. Let $m \geq 1$ be a fixed integer. A sequence of random variables $\left\{X_{n}, n \geq 1\right\}$ is said to be $m-P N Q D$ if for all positive integers $j$ and $k$ with $|j-k| \geq m, X_{j}$ and $X_{k}$ are $N Q D$.

Clearly, PNQD is the special case $m=1$ of the concept of $m$-PNQD. Indeed, if $\left\{X_{n}, n \geq 1\right\}$ is $m$-PNQD for some $m \geq 1$, then $\left\{X_{n}, n \geq 1\right\}$ is $m^{\prime}$-PNQD for all $m^{\prime}>m$.

Li et al. ([13]) showed that for every sequence of continuous distribution functions $\left\{F_{n}, n \geq 1\right\}$, a sequence of PNQD random variables $\left\{X_{n}, n \geq 1\right\}$ can be constructed such that the distribution function of $X_{n}$ is $F_{n}, n \geq 1$ and such that for all $k \geq 1,\left\{X_{n}, n \geq k\right\}$ is not a sequence of independent random variables.

We now provide two examples of sequences of $m$-PNQD random variables.

EXAMPLE 1.3. Let $\left\{Y_{n}, n \geq 1\right\}$ be a PNQD sequence of random variables and let $m \geq 2$. For $n \geq 1$, let $r \geq 1$ be such that $(r-1) m+1 \leq n \leq r m$ and let $X_{n}=Y_{r}$. Then $\left\{X_{n}, n \geq 1\right\}$ is a sequence of $m$-PNQD random variables.

EXAMPLE 1.4. Let $\left\{Y_{n}, n \geq 1\right\}$ be a PNQD sequence of random variables, let $m \geq 2$, and let $\left\{Z_{i j}, 1 \leq j \leq m-1, i \geq 1\right\}$ be an array of random variables such that for all $i \neq i^{\prime}$ and all $1 \leq j, j^{\prime} \leq m-1, Y_{i}$ and $Z_{i^{\prime} j^{\prime}}$ are NQD and $Z_{i j}$ and $Z_{i^{\prime} j^{\prime}}$ are NQD. For $n \geq 1$, let $r \geq 1$ be such that $(r-1) m+1 \leq n \leq r m$ and let

$$
X_{n}= \begin{cases}Y_{n}, & \text { if } n=(r-1) m+1, \\ Z_{r, n-(r-1) m-1}, & \text { if }(r-1) m+2 \leq n \leq r m .\end{cases}
$$

Then $\left\{X_{n}, n \geq 1\right\}$ is a sequence of $m$-PNQD random variables.

Years after the appearance of Lehmann ([12]), a large literature of investigation concerning the convergence properties of PNQD random variables has emerged. We refer the reader to Matula ([16]), Qi ([21]), Patterson and Taylor ([19]), Kim and Kim ([10]), Kim and Kim ([11]), Taylor et al. ([25]), Wu ([27]), Ordóñez Cabrera and Volodin ([18]), Li et al. ([13]), Gan and Chen $([6])$, Li and Yang ([14]), Meng and Lin ([17]), Gerasimov ([7]), Xing ([31]), 
Wu and Jiang ([28]), Wu and Guan ([29]), Wu and Wang ([30]), and Sung ([22]) among others.

The purpose of this article is to investigate complete convergence and the strong law of large numbers (SLLN) for sequences of $m$-PNQD random variables.

The concept of the complete convergence was introduced by Hsu and Robbins ([8]). A sequence of random variables $\left\{U_{n}, n \geq 1\right\}$ is said to converge completely to a constant $\theta$ if

$$
\sum_{n=1}^{\infty} P\left(\left|U_{n}-\theta\right|>\varepsilon\right)<\infty \text { for all } \varepsilon>0 .
$$

In view of the Borel-Cantelli lemma, the above result implies that $U_{n} \rightarrow \theta$ almost surely (a.s.). Therefore, complete convergence is an important tool which is often used in establishing a.s. convergence of sums of random variables.

Choi and Sung ([4]) studied the SLLN for sequences of pairwise independent random variables and obtained the following Marcinkiewicz-Zygmund type result.

TheOREM 1.5. Let $\left\{X_{n}, n \geq 1\right\}$ be a sequence of pairwise independent random variables. Suppose that $\left\{X_{n}, n \geq 1\right\}$ is stochastically dominated by a random variable $X$ (this technical definition is given in the next section). If

$$
E\left(|X|^{r}\left(\log ^{+}|X|\right)^{2}\right)<\infty \quad \text { for some } 1<r<2,
$$

then

$$
\lim _{n \rightarrow \infty} n^{-1 / r} \sum_{k=1}^{n}\left(X_{k}-E X_{k}\right)=0 \quad \text { a.s. }
$$

Hu et al. ([9]) also studied the SLLN for sequences of PNQD random variables and obtained the following Marcinkiewicz-Zygmund type result.

THEOREM 1.6. Let $\left\{X_{n}, n \geq 1\right\}$ be a sequence of $P N Q D$ random variables with $E X_{n}=0$ for all $n \geq 1$. Suppose that $\left\{X_{n}, n \geq 1\right\}$ is stochastically dominated by a random variable $X$. If there exist constants $1 \leq r<2$ and $\alpha>r+1$ such that

$$
E\left(|X|^{r}\left(\log ^{+}|X|\right)^{\alpha}\right)<\infty,
$$

then

$$
\lim _{n \rightarrow \infty} n^{-1 / r} \sum_{k=1}^{n} X_{k}=0 \quad \text { a.s. }
$$

If we assume that $E X_{k}=0$ for all $k \geq 1$ in Theorem 1.5, then (1.2) is exactly (1.4). To some extent, Theorem 1.6 extends Theorem 1.5 from the 
pairwise independent case to the PNQD case. However, when $r>1$, the moment condition (1.3) is not optimal since it is stronger than (1.1).

In the current work, we obtain some results on complete convergence and the Marcinkiewicz-Zygmund SLLN for sequences of $m$-PNQD random variables which improve and extend Theorems 1.5 and 1.6 to the $m$-PNQD case. We also establish the Kolmogorov SLLN for sequences of $m$-PNQD random variables. We point out that the method used in this article differs from those in Choi and Sung ([4]) and $\mathrm{Hu}$ et al. ([9]).

Throughout this paper, the symbol $C$ is used to represent positive constants whose values may change from one place to another.

\section{Preliminaries}

To prove our main results, we need the following technical lemmas.

Lemma 2.1 ([12]). Let $X$ and $Y$ be NQD random variables. Then

(i) $\operatorname{Cov}(X, Y) \leq 0$,

(ii) If $f$ and $g$ are Borel functions which are both monotone increasing (or both monotone decreasing), then $f(X)$ and $g(Y)$ are $N Q D$.

LEMMA $2.2([27])$. Let $\left\{X_{n}, n \geq 1\right\}$ be a sequence of PNQD random variables with mean zero and $E X_{n}^{2}<\infty, n \geq 1$, and let $T_{j}(k)=\sum_{i=j+1}^{j+k} X_{i}$, $j \geq 0, k \geq 1$. Then

$$
E\left(\max _{1 \leq k \leq n}\left(T_{j}(k)\right)^{2}\right) \leq C\left(\log ^{2} n\right) \sum_{i=j+1}^{j+n} E X_{i}^{2}, \quad n \geq 1
$$

where $\log n=\log _{e} \max \{e, n\}, n \geq 1$.

LEMMA 2.3. Let $\left\{X_{n}, n \geq 1\right\}$ be a sequence of $m-P N Q D$ random variables with mean zero and $E X_{n}^{2}<\infty, n \geq 1$. Then there exists a positive constant $C$ depending only on $m$ such that

$$
E\left(\max _{1 \leq j \leq n}\left(\sum_{k=1}^{j} X_{k}\right)^{2}\right) \leq C\left(\log ^{2} 2 n\right) \sum_{k=1}^{n} E X_{k}^{2}, \quad n \geq 1 .
$$

where $\log n=\log _{e} \max \{e, n\}, n \geq 1$.

Proof. It is clear that exists a suitably chosen $C$ satisfying the inequality in the conclusion for $1 \leq n \leq m$ since this set of values of $n$ is finite. Therefore, we only need to consider the case $n>m$. Given any $1 \leq j \leq n$, let $\nu=\left[\frac{j}{m}\right]$ and $\tau=\left[\frac{n}{m}\right]$. Define

$$
V_{k}^{(j)}=\left\{\begin{array}{ll}
X_{k}, & \text { if } 1 \leq k \leq j \\
0, & \text { if } k>j
\end{array} \text { for } 1 \leq j \leq n\right.
$$


and

$$
T_{j l}=\sum_{i=0}^{\nu} V_{m i+l}^{(j)} \text { for } 1 \leq l \leq m .
$$

Clearly $\sum_{k=1}^{j} X_{k}=\sum_{l=1}^{m} T_{j l}=\sum_{l=1}^{m} \sum_{i=0}^{\nu} V_{m i+l}^{(j)}$. Hence by the $C_{r^{-}}$ inequality and Lemma 2.2 ,

$$
\begin{aligned}
& E\left(\max _{1 \leq j \leq n}\left(\sum_{k=1}^{j} X_{k}\right)^{2}\right) \\
& \quad \leq E\left(\max _{1 \leq j \leq n}\left(\sum_{l=1}^{m} T_{j l}\right)^{2}\right) \leq m \sum_{l=1}^{m} E\left(\max _{1 \leq j \leq n} T_{j l}^{2}\right) \\
& \quad \leq m \sum_{l=1}^{m} E\left(\max _{0 \leq \nu \leq \tau}\left(\sum_{i=0}^{\nu} V_{m i+l}^{(j)}\right)^{2}\right) \leq C m \sum_{l=1}^{m}\left(\log ^{2} 2 \tau\right) \sum_{i=0}^{\tau} E\left(V_{m i+l}^{(n)}\right)^{2} \\
& \quad=C\left(\log ^{2} 2 n\right) \sum_{l=1}^{m} \sum_{i=0}^{\tau} E\left(V_{m i+l}^{(n)}\right)^{2}=C\left(\log ^{2} 2 n\right) \sum_{k=1}^{n} E X_{k}^{2} .
\end{aligned}
$$

The proof is completed.

LEMMA $2.4([3])$. Let $\left\{X_{n}, n \geq 1\right\}$ be a sequence of nonnegative random variables with $\operatorname{Var}\left(X_{n}\right)<\infty, n \geq 1$ and let $\{f(n), n \geq 1\}$ be a sequence such that $0<f(n) \uparrow \infty$. Assume that

(i) $\sup _{n \geq 1} f^{-1}(n) \sum_{k=1}^{n} E X_{k}<\infty$;

(ii) there is a double sequence $\rho_{i j}$ of nonnegative real numbers such that

$$
\operatorname{Var}\left(\sum_{k=1}^{n} X_{k}\right) \leq \sum_{i=1}^{n} \sum_{j=1}^{n} \rho_{i j} \text { for each } n \geq 1
$$

(iii) $\sum_{i=1}^{\infty} \sum_{j=1}^{\infty} \frac{\rho_{i j}}{f^{2}(i \vee j)}<\infty$, where $i \vee j=\max (i, j), i \geq 1, j \geq 1$.

Then

$$
f^{-1}(n) \sum_{k=1}^{n}\left(X_{k}-E X_{k}\right) \rightarrow 0 \quad \text { a.s. as } n \rightarrow \infty .
$$

The following concept of stochastic domination is a generalization of the concept of identical distributions. A sequence of random variables $\left\{X_{n}, n \geq\right.$ $1\}$ is said to be stochastically dominated by a random variable $X$ if there exists a constant $C>0$ such that

$$
\sup _{n \geq 1} P\left(\left|X_{n}\right|>x\right) \leq C P(|X|>x) \text { for all } x \geq 0 \text {. }
$$

Stochastic dominance is of course automatic with $X=X_{1}$ and $C=1$ if $\left\{X_{n}, n \geq 1\right\}$ is a sequence of identically distributed random variables. It 
follows from Lemma 5.2.2 of Taylor ([24], p. 123) (or Lemma 3 of [26]) that stochastic dominance of a sequence of random variables can be accomplished by the random variables in the sequence having a bounded absolute $r$-th moment $(r>0)$. Specifically, if $\sup _{n>1} E\left|X_{n}\right|^{r}<\infty$ for some $r>0$, then there exists a random variable $X$ with $E|X|^{s}<\infty$ for all $0<s<r$ such that (2.1) holds with $C=1$. (The provision that $r>1$ in Lemma 5.2.2 of Taylor $([24$, p. 123]) (or [26, Lemma 3]) is not needed as was pointed out by Adler et al. $([1]))$.

Lemma 2.5. Let $\left\{X_{n}, n \geq 1\right\}$ be a sequence of random variables which is stochastically dominated by a random variable $X$. Then there exists a constant $C$ such that, for all $q>0$ and $x>0$,

(i) $E\left(\left|X_{k}\right|^{q} I\left(\left|X_{k}\right| \leq x\right)\right) \leq C\left\{E\left(|X|^{q} I(|X| \leq x)\right)+x^{q} P(|X|>x)\right\}$,

(ii) $E\left(\left|X_{k}\right|^{q} I\left(\left|X_{k}\right|>x\right)\right) \leq C E\left(|X|^{q} I(|X|>x)\right)$.

This lemma can be easily proved by using integration by parts. We omit the details.

\section{MAin RESUlts}

Now we present our main results and their proofs.

THEOREM 3.1. Let $\left\{X_{n}, n \geq 1\right\}$ be a sequence of $m-P N Q D$ random variables which is stochastically dominated by a random variable $X$. If (1.1) holds, then for all $\varepsilon>0$,

$$
\sum_{n=1}^{\infty} n^{-1} P\left(\max _{1 \leq j \leq n}\left|\sum_{k=1}^{j}\left(X_{k}-E X_{k}\right)\right|>n^{1 / r} \varepsilon\right)<\infty .
$$

Proof. For fixed $n \geq 1$, let

$$
\begin{aligned}
& Y_{n k}=-n^{1 / r} I\left(X_{k}<-n^{1 / r}\right)+X_{k} I\left(\left|X_{k}\right| \leq n^{1 / r}\right)+n^{1 / r} I\left(X_{k}>n^{1 / r}\right), \\
& Z_{n k}=\left(X_{k}+n^{1 / r}\right) I\left(X_{k}<-n^{1 / r}\right)+\left(X_{k}-n^{1 / r}\right) I\left(X_{k}>n^{1 / r}\right) .
\end{aligned}
$$

Then $Y_{n k}+Z_{n k}=X_{k}$, and it follows by the definition of $m$-PNQD and Lemma 2.1 (ii) that $\left\{Y_{n k}, k \geq 1\right\}$ is a sequence of $m$-PNQD random variables. Then

$$
\begin{aligned}
& \sum_{n=1}^{\infty} n^{-1} P\left(\max _{1 \leq j \leq n}\left|\sum_{k=1}^{j}\left(X_{k}-E X_{k}\right)\right|>n^{1 / r} \varepsilon\right) \\
& \leq \sum_{n=1}^{\infty} n^{-1} P\left(\max _{1 \leq j \leq n}\left|\sum_{k=1}^{j}\left(Z_{n k}-E Z_{n k}\right)\right|>n^{1 / r} \varepsilon / 2\right) \\
& \quad+\sum_{n=1}^{\infty} n^{-1} P\left(\max _{1 \leq j \leq n}\left|\sum_{k=1}^{j}\left(Y_{n k}-E Y_{n k}\right)\right|>n^{1 / r} \varepsilon / 2\right) \\
& =: I_{1}+I_{2} .
\end{aligned}
$$


To prove (3.1), it only needs to be shown that $I_{1}<\infty$ and $I_{2}<\infty$. Note that $\left|Z_{n k}\right| \leq\left|X_{k}\right| I\left(\left|X_{k}\right|>n^{1 / r}\right)$. By the Markov inequality, Lemma 2.5(ii) and (1.1), we have

$$
\begin{aligned}
I_{1} & \leq C \sum_{n=1}^{\infty} n^{-1-1 / r} \sum_{k=1}^{n} E\left|Z_{n k}-E Z_{n k}\right| \\
& \leq C \sum_{n=1}^{\infty} n^{-1-1 / r} \sum_{k=1}^{n} E\left(\left|X_{k}\right| I\left(\left|X_{k}\right|>n^{1 / r}\right)\right) \\
& \leq C \sum_{n=1}^{\infty} n^{-1 / r} \sum_{s=n}^{\infty} E\left(|X| I\left(s<|X|^{r} \leq s+1\right)\right) \\
& =C \sum_{s=1}^{\infty} E\left(|X| I\left(s<|X|^{r} \leq s+1\right)\right) \sum_{n=1}^{s} n^{-1 / r} \\
& \leq C \sum_{s=1}^{\infty} s^{1-1 / r} E\left(|X| I\left(s<|X|^{r} \leq s+1\right)\right) \leq C E|X|^{r}<\infty .
\end{aligned}
$$

Next we prove $I_{2}<\infty$. By the Markov inequality, Lemma 2.3, and Lemma 2.5(i), we have

$$
\begin{aligned}
I_{2} \leq & C \sum_{n=1}^{\infty} n^{-1-2 / r}\left(\log ^{2} n\right) \sum_{k=1}^{n} E Y_{n k}^{2} \\
\leq & C \sum_{n=1}^{\infty} n^{-1-2 / r}\left(\log ^{2} n\right) \sum_{k=1}^{n} E\left(X_{k}^{2} I\left(\left|X_{k}\right| \leq n^{1 / r}\right)\right) \\
& +C \sum_{n=1}^{\infty} n^{-1}\left(\log ^{2} n\right) \sum_{k=1}^{n} P\left(\left|X_{k}\right|>n^{1 / r}\right) \\
\leq & C \sum_{n=1}^{\infty} n^{-2 / r}\left(\log ^{2} n\right) E\left(X^{2} I\left(|X| \leq n^{1 / r}\right)\right) \\
& +C \sum_{n=1}^{\infty}\left(\log ^{2} n\right) P\left(|X|>n^{1 / r}\right) \\
= & : I_{3}+I_{4} .
\end{aligned}
$$


Since the function $\log ^{2} x$ is slowly varying at $\infty$, by applying Theorem VIII. 9.1 of Feller ([5, p. 281]), we have

$$
\begin{aligned}
I_{3} & =C \sum_{n=1}^{\infty} n^{-2 / r}\left(\log ^{2} n\right) \sum_{s=1}^{n} E\left(X^{2} I\left(s-1<|X|^{r} \leq s\right)\right) \\
& =C \sum_{s=1}^{\infty} E\left(X^{2} I\left(s-1<|X|^{r} \leq s\right)\right) \sum_{n=s}^{\infty} n^{-2 / r} \log ^{2} n \\
& \leq C \sum_{s=1}^{\infty} s^{1-2 / r}\left(\log ^{2} s\right) E\left(X^{2} I\left(s-1<|X|^{r} \leq s\right)\right) \\
& \leq C E\left(|X|^{r}\left(\log ^{+}|X|\right)^{2}\right)<\infty
\end{aligned}
$$

and

$$
\begin{aligned}
I_{4} & \leq C \sum_{n=1}^{\infty} n^{-1 / r}\left(\log ^{2} n\right) E\left(|X| I\left(|X|>n^{1 / r}\right)\right) \\
& =C \sum_{n=1}^{\infty} n^{-1 / r}\left(\log ^{2} n\right) \sum_{s=n}^{\infty} E\left(|X| I\left(s<|X|^{r} \leq s+1\right)\right) \\
& =C \sum_{s=1}^{\infty} E\left(|X| I\left(s<|X|^{r} \leq s+1\right)\right) \sum_{n=1}^{s} n^{-1 / r} \log ^{2} n \\
& \leq C \sum_{s=1}^{\infty} s^{1-1 / r}\left(\log ^{2} s\right) E\left(|X| I\left(s<|X|^{r} \leq s+1\right)\right) \\
& \leq C E\left(|X|^{r}\left(\log ^{+}|X|\right)^{2}\right)<\infty .
\end{aligned}
$$

The proof is completed.

The following corollary provides a Marcinkiewicz-Zygmund type SLLN for a sequence of $m$-PNQD random variables. Corollary 3.2 is indeed a weaker version of Corollary 2.2 in [2] (see Remark 3.7 below).

Corollary 3.2. Under the conditions of Theorem 3.1, (1.2) holds.

Proof. Let $S_{j}=\sum_{k=1}^{j}\left(X_{k}-E X_{k}\right), j \geq 1$. From (3.1), we have for arbitrary $\varepsilon>0$,

$$
\begin{aligned}
\infty & >\sum_{n=1}^{\infty} n^{-1} P\left(\max _{1 \leq j \leq n}\left|S_{j}\right|>n^{\frac{1}{r}} \varepsilon\right) \\
& =\sum_{i=0}^{\infty} \sum_{n=2^{i}}^{2^{i+1}-1} n^{-1} P\left(\max _{1 \leq j \leq n}\left|S_{j}\right|>n^{\frac{1}{r}} \varepsilon\right) \\
& \geq \frac{1}{2} \sum_{i=1}^{\infty} P\left(\max _{1 \leq j \leq 2^{i}}\left|S_{j}\right|>2^{\frac{i+1}{r}} \varepsilon\right) .
\end{aligned}
$$


Then by the Borel-Cantelli Lemma and the arbitrariness of $\varepsilon>0$,

$$
\lim _{i \rightarrow \infty} 2^{-\frac{i+1}{r}} \max _{1 \leq j \leq 2^{i}}\left|S_{j}\right|=0 \quad \text { a.s. }
$$

For every positive integer $n$, there exists a positive integer $i_{0}$ such that $2^{i_{0}-1} \leq$ $n<2^{i_{0}}$. Then $i_{0} \rightarrow \infty$ as $n \rightarrow \infty$ and

$$
\begin{aligned}
n^{-\frac{1}{r}}\left|S_{n}\right| & \leq \max _{2^{i_{0}-1} \leq j<2^{i_{0}}} j^{-\frac{1}{r}}\left|S_{j}\right| \\
& \leq 2^{\frac{2}{r}} 2^{-\frac{i_{0}+1}{r}} \max _{1 \leq j<2^{i_{0}}}\left|S_{j}\right| \rightarrow 0 \text { a.s. as } n \rightarrow \infty .
\end{aligned}
$$

The proof is completed.

REMARK 3.3. Since pairwise independence implies $m$-PNQD and since (3.1) implies (1.2) as has been shown in the proof of Corollary 3.2, Theorem 3.1 and Corollary 3.2 improve and extend Theorem 1.5. In addition, since PNQD implies $m$-PNQD and since (1.1) is weaker than (1.3) when $r>1$, Theorem 3.1 and Corollary 3.2 also improve and extend Theorem 1.6.

REMARK 3.4. Martikainen ([15]) showed for a sequence of pairwise i.i.d. random variables that (1.2) holds under a weaker moment condition than that in Theorem 1.5. He proved that the condition (1.1) can be improved to $E\left|X_{1}\right|^{r}\left(\log \left|X_{1}\right|\right)^{\tau}<\infty$ for $1<r<2$ and $\tau>\max \{0,4 r-6\}$. Sung ([23]) improved the result of Martikainen ([15]) under the much weaker moment condition $E\left|X_{1}\right|^{r}\left(\log \log \left|X_{1}\right|\right)^{2(r-1)}<\infty$ where $1<r<2$. We find that the truncation method used in Sung ([23]) is not suitable for obtaining PNQD or $m$-PNQD results. Therefore, it is an open problem as to whether or not Theorem 3.1 and Corollary 3.2 hold with Sung's ([23]) weaker moment condition.

The next theorem provides a Kolmogorov type SLLN for a sequence of $m$-PNQD random variables.

THEOREM 3.5. Let $\left\{X_{n}, n \geq 1\right\}$ be a sequence of $m$-PNQD random variables which is stochastically dominated by a random variable $X$. If $E\left(|X| \log ^{+}|X|\right)<\infty$, then

$$
\lim _{n \rightarrow \infty} n^{-1} \sum_{i=1}^{n}\left(X_{k}-E X_{k}\right) \rightarrow 0 \quad \text { a.s. }
$$

Proof. Without loss of generality, we may assume that $E X_{k}=0, k \geq 1$. For $k \geq 1$, let

$$
\begin{aligned}
Y_{k} & =-k I\left(X_{k}<-k\right)+X_{k} I\left(\left|X_{k}\right| \leq k\right)+k I\left(X_{k}>k\right), \\
Y_{k}^{*} & =\left(X_{k}+k\right) I\left(X_{k}<-k\right)+\left(X_{k}-k\right) I\left(X_{k}>k\right) .
\end{aligned}
$$


Then it follows by the definition of $m$-PNQD and Lemma 2.1(ii) that $\left\{Y_{k}, k \geq\right.$ $1\}$ is a sequence of $m$-PNQD random variables. From $E|X|<\infty$, we have

$$
\sum_{n=1}^{\infty} P\left(X_{n} \neq Y_{n}\right)=\sum_{n=1}^{\infty} P\left(\left|X_{n}\right|>n\right) \leq C \sum_{n=1}^{\infty} P(|X|>n) \leq C E|X|<\infty .
$$

Thus by the Borel-Cantelli lemma, we have

$$
n^{-1} \sum_{k=1}^{n}\left(X_{k}-Y_{k}\right) \rightarrow 0 \quad \text { a.s. } n \rightarrow \infty .
$$

Note that $\left|Y_{k}^{*}\right| \leq\left|X_{k}\right| I\left(\left|X_{k}\right|>k\right), k \geq 1$. By $E X_{k}=0, k \geq 1$ and Lemma 2.5(ii), we have

$$
\begin{aligned}
\sum_{k=1}^{\infty} k^{-1}\left|E Y_{k}\right| & =\sum_{k=1}^{\infty} k^{-1}\left|E Y_{k}^{*}\right| \leq C \sum_{k=1}^{\infty} k^{-1} E(|X| I(|X|>k)) \\
& =C \sum_{k=1}^{\infty} k^{-1} \sum_{j=k}^{\infty} E(|X| I(j<|X| \leq j+1)) \\
& =C \sum_{j=1}^{\infty} E(|X| I(j<|X| \leq j+1)) \sum_{k=1}^{j} k^{-1} \\
& \leq C \sum_{j=1}^{\infty}(\log j) E(|X| I(j<|X| \leq j+1)) \\
& \leq C E\left(|X| \log ^{+}|X|\right)<\infty .
\end{aligned}
$$

Hence by the Kronecker lemma, we have

$$
n^{-1} \sum_{k=1}^{n}\left|E Y_{k}\right| \rightarrow 0 \quad \text { a.s. } n \rightarrow \infty,
$$

which implies

$$
n^{-1} \sum_{k=1}^{n} E Y_{k} \rightarrow 0 \quad \text { a.s. } n \rightarrow \infty .
$$

Next, we will prove that

$$
n^{-1} \sum_{k=1}^{n}\left(Y_{k}-E Y_{k}\right) \rightarrow 0 \quad \text { a.s. } n \rightarrow \infty .
$$

Let $Z_{k}=\left(Y_{k}-E Y_{k}\right)^{+}, R_{k}=\left(Y_{k}-E Y_{k}\right)^{-}, k \geq 1$, and $f(n)=n, n \geq 1$, where $x^{+}=\max \{x, 0\}$ and $x^{-}=\max \{-x, 0\}$. By the definition of $m$-PNQD and Lemma 2.1(ii), $\left\{Z_{k}, k \geq 1\right\}$ and $\left\{R_{k}, k \geq 1\right\}$ are each $m$-PNQD sequences. 
Now by Lemma 2.5(i), we have

$$
\begin{aligned}
& \sup _{n \geq 1} n^{-1} \sum_{k=1}^{n} E Z_{k} \leq \sup _{n \geq 1} n^{-1} \sum_{k=1}^{n} E\left|Y_{k}-E Y_{k}\right| \\
& \leq C \sup _{n \geq 1} n^{-1} \sum_{k=1}^{n} E(|X| I(|X| \leq k))+C \sum_{k=1}^{n} P(|X|>k) \\
& \leq C E|X|<\infty .
\end{aligned}
$$

Define

$$
\rho_{i j}=\left\{\begin{array}{cl}
\operatorname{Var}\left(Z_{i}\right), & \text { if } j=i, \\
0, & \text { if } j \neq i .
\end{array}\right.
$$

Given any $1 \leq k \leq n$, take $\tau=\left[\frac{n}{m}\right]$. Let

$$
Z_{k}^{*}= \begin{cases}Z_{k}, & \text { if } 1 \leq k \leq n, \\ 0, & \text { if } k>n .\end{cases}
$$

Clearly $\sum_{k=1}^{n} Z_{k}=\sum_{j=1}^{m} \sum_{i=0}^{\tau} Z_{m i+j}^{*}$. Therefore,

$$
\begin{aligned}
\operatorname{Var}\left(\sum_{k=1}^{n} Z_{k}\right) & =\operatorname{Var}\left(\sum_{j=1}^{m} \sum_{i=0}^{\tau} Z_{m i+j}^{*}\right) \\
& =\sum_{j=1}^{m} \operatorname{Var}\left(\sum_{i=0}^{\tau} Z_{m i+j}^{*}\right)+2 \sum_{1 \leq l<s \leq m} \operatorname{Cov}\left(\sum_{i=0}^{\tau} Z_{m i+l}^{*}, \sum_{t=0}^{\tau} Z_{m t+s}^{*}\right) \\
& =: A+B .
\end{aligned}
$$

For $A$, by the definition of $m$-PNQD and Lemma 2.1(i), we have

$$
\begin{aligned}
A & =\sum_{j=1}^{m}\left(\sum_{i=0}^{\tau} \operatorname{Var}\left(Z_{m i+j}^{*}\right)+2 \sum_{0 \leq u<v \leq \tau} \operatorname{Cov}\left(Z_{m u+j}^{*}, Z_{m v+j}^{*}\right)\right) \\
& \leq \sum_{j=1}^{m} \sum_{i=0}^{\tau} \operatorname{Var}\left(Z_{m i+j}^{*}\right)=\sum_{k=1}^{n} \operatorname{Var}\left(Z_{k}\right) .
\end{aligned}
$$

For $B$, by a property of covariance, the definition of $m$-PNQD, and Lemma 2.1(i), we have

$$
B=2 \sum_{1 \leq l<s \leq m} \sum_{i=0}^{\tau} \sum_{t=0}^{\tau} \operatorname{Cov}\left(Z_{m i+l}^{*}, Z_{m t+s}^{*}\right) \leq 0 .
$$

Hence we get

$$
\operatorname{Var}\left(\sum_{k=1}^{n} Z_{k}\right) \leq \sum_{k=1}^{n} \operatorname{Var}\left(Z_{k}\right)=\sum_{k=1}^{n} \sum_{j=1}^{n} \rho_{k j}, \quad n \geq 1 .
$$


Note that

$$
\begin{aligned}
& \sum_{k=1}^{\infty} k^{-2} E\left(X^{2} I(|X| \leq k)\right) \\
& \quad=\sum_{k=1}^{\infty} k^{-2} \sum_{s=1}^{k} E\left(X^{2} I(s-1<|X| \leq s)\right) \\
& \quad=\sum_{s=1}^{\infty} E\left(X^{2} I(s-1<|X| \leq s)\right) \sum_{k=s}^{\infty} k^{-2} \\
& \quad \leq \sum_{s=1}^{\infty} s^{-1} E\left(X^{2} I(s-1<|X| \leq s)\right) \leq E|X| .
\end{aligned}
$$

Then by the definitions of $\rho_{i j}$ and $f(n)$, we have by Lemma 2.5(i) that

$$
\begin{aligned}
\sum_{k=1}^{\infty} \sum_{j=1}^{\infty} \frac{\rho_{k j}}{f^{2}(k \vee j)} & =\sum_{k=1}^{\infty} \frac{\operatorname{Var}\left(Z_{k}\right)}{k^{2}} \leq \sum_{k=1}^{\infty} \frac{E Y_{k}^{2}}{k^{2}} \\
& =\sum_{k=1}^{\infty} k^{-2}\left(E\left(X_{k}^{2} I\left(\left|X_{k}\right| \leq k\right)\right)+k^{2} P\left(\left|X_{k}\right|>k\right)\right) \\
& \leq C \sum_{k=1}^{\infty} k^{-2} E\left(X^{2} I(|X| \leq k)\right)+C \sum_{k=1}^{\infty} P(|X|>k) \\
& \leq 2 E|X|<\infty .
\end{aligned}
$$

In view of (3.6), (3.7) and (3.8), we get by Lemma 2.4 that

$$
n^{-1} \sum_{k=1}^{n}\left(Z_{k}-E Z_{k}\right) \rightarrow 0 \quad \text { a.s. } n \rightarrow \infty .
$$

An argument similar to that for establishing (3.9) gives

$$
n^{-1} \sum_{k=1}^{n}\left(R_{k}-E R_{k}\right) \rightarrow 0 \quad \text { a.s. } n \rightarrow \infty .
$$

By (3.9) and (3.10), we get (3.5). Combining (3.3), (3.4) and (3.5) yields (3.2). The proof is completed.

REMARK 3.6. If $m=1$, then the moment condition $E\left(\left|X_{1}\right| \log ^{+}\left|X_{1}\right|\right)<$ $\infty$ can be weakened to $E\left|X_{1}\right|<\infty$ for a sequence $\left\{X_{n}, n \geq 1\right\}$ of PNQD identically distributed random variables as was proved by Matula ([16]). Matula's ([16]) result was extended by $\mathrm{Li}$ et al. ([13]) to the case of weighted sums of PNQD identically distributed random variables as a consequence of a much more general result they obtained in the same article. It remains open as to whether or not the moment condition $E\left(|X| \log ^{+}|X|\right)<\infty$ can be weakened to $E|X|<\infty$ in Theorem 3.5. 
REMARK 3.7. Some discussion is in order comparing our work with that of Anh ([2]). Theorem 2.1 of Anh ([2]) is a Marcinkiewicz-Zygmund type SLLN for a sequence of blockwise and $m$-PNQD random variables with respect to a sequence of blocks $\left\{B_{k}, k \geq 1\right\}$. The blocks are disjoint finite sets of positive integers and each block is an index set for a finite set of random variables. As we mentioned prior to Definition 1.2 above, it was Anh ([2]) who introduced the $m$-PNQD structure for a finite set of random variables. Anh ([2]) did not impose any dependence conditions between random variables with indices in different blocks. In our Theorem 3.1 and Corollary 3.2, we establish, respectively, a complete convergence theorem and a Marcinkiewicz-Zygmund type SLLN under a moment condition which is identical to that of Theorem 2.1 and Corollary 2.2 of Anh ([2]) when $1<r<2$. Since a sequence of $m$-PNQD random variables is automatically a sequence of blockwise and $m$-PNQD random variables with respect to the sequence of blocks $\left\{\left[2^{k-1}, 2^{k}\right), k \geq 1\right\}$, our Corollary 3.2 indeed follows immediately from Corollary 2.2 of Anh ([2]) but their proofs are entirely different. On the other hand, the Kolmogorov type SLLN provided by our Theorem 3.5 has a moment condition which is strictly weaker than that of Corollary 2.2 of Anh ([2]) when $r=1$.

\section{ACKNOWLEDGEMENTS.}

The authors are grateful to the referee for carefully reading the manuscript and for offering comments which enabled them to substantially improve the paper. In particular, the referee called to the authors' attention the article by Anh ([2]). The research of Y. Wu was supported by the $\mathrm{Hu}-$ manities and Social Sciences Foundation for the Youth Scholars of Ministry of Education of China (12YJCZH217), the Natural Science Foundation of Anhui Province (1308085MA03), the key Grant Project for Backup Academic Leaders of Tongling University (2014tlxyxs21), and the Key NSF of Anhui Educational Committee (KJ2014A255).

\section{REFERENCES}

[1] A. Adler, A. Rosalsky and R. L. Taylor, Some strong laws of large numbers for sums of random elements, Bull. Inst. Math. Acad. Sinica 20 (1992), 335-357.

[2] V. T. N. Anh, A strong limit theorem for sequences of blockwise and pairwise negative quadrant $m$-dependent random variables, Bull. Malays. Math. Sci. Soc. (2) 36 (2013), 159-164.

[3] T. K. Chandra and A. Goswami, Cesàro uniform integrability and the strong law of large numbers, Sankhyā Ser. A 54 (1992), 215-231.

[4] B. D. Choi and S. H. Sung, On convergence of $\left(S_{n}-E S_{n}\right) / n^{1 / r}, 1<r<2$, for pairwise independent random variables, Bull. Korean Math. Soc. 22 (1985), 79-82.

[5] W. Feller, An introduction to probability theory and its applications. Vol. II, 2nd ed., John Wiley, New York, 1971.

[6] S. Gan and P. Chen, Some limit theorems for sequences of pairwise NQD random variables, Acta Math. Sci. Ser. B Engl. Ed. 28 (2008), 269-281. 
[7] M. Y. Gerasimov, The strong law of large numbers for pairwise negatively dependent random variables, Vestnik Moskov. Univ. Ser. XV Vychisl. Mat. Kibernet. 2009 (2009), 7-13 (in Russian). English translation in: Moscow Univ. Comput. Math. Cybernet. 33 (2009), 51-58.

[8] P. L. Hsu and H. Robbins, Complete convergence and the law of large numbers, Proc. Nat. Acad. Sci. U. S. A. 33 (1947), 25-31.

[9] S. Hu, X. Liu, X. Wang and X. Li, Strong law of large numbers of partial sums for pairwise NQD sequences, J. Math. Res. Appl. 33 (2013), 111-116.

[10] T.-S. Kim and H.-C. Kim, On the weak law of large numbers for weighted sums of pairwise negative quadrant dependent random variables, Int. J. Math. Game Theory Algebra 10 (2000), 473-482.

[11] T.-S. Kim and H.-C. Kim, On the law of large numbers for weighted sums of pairwise negatively quadrant dependent random variables, Bull. Korean Math. Soc. 38 (2001), 55-63.

[12] E. L. Lehmann, Some concepts of dependence, Ann. Math. Statist. 37 (1966), 11371153.

[13] D. Li, A. Rosalsky and A. I. Volodin, On the strong law of large numbers for sequences of pairwise negative quadrant dependent random variables, Bull. Inst. Math. Acad. Sin. (N.S.) 1 (2006), 281-305.

[14] R. Li and W. Yang, Strong convergence of pairwise NQD random sequences, J. Math. Anal. Appl. 344 (2008), 741-747.

[15] A. Martikainen, On the strong law of large numbers for sums of pairwise independent random variables, Statist. Probab. Lett. 25 (1995), 21-26.

[16] P. Matula, A note on the almost sure convergence of sums of negatively dependent random variables, Statist. Probab. Lett. 15 (1992), 209-213.

[17] Y. Meng and Z. Lin, On the weak laws of large numbers for arrays of random variables, Statist. Probab. Lett. 79 (2009), 2405-2414.

[18] M. Ordóñez Cabrera and A. Volodin, Mean convergence theorems and weak laws of large numbers for weighted sums of random variables under a condition of weighted integrability, J. Math. Anal. Appl. 305 (2005), 644-658.

[19] R. F. Patterson and R. L. Taylor, Strong laws of large numbers for negatively dependent random elements, Nonlinear Anal. 30 (1997), 4229-4235.

[20] R. Pemantle, Towards a theory of negative dependence, J. Math. Phys. 41 (2000), 1371-1390.

[21] Y. C. Qi, Limit theorems for sums and maxima of pairwise negative quadrant dependent random variables, Systems Sci. Math. Sci. 8 (1995), 249-253.

[22] S. H. Sung, Convergence in $r$-mean of weighted sums of NQD random variables, Appl. Math. Lett. 26 (2013), 18-24.

[23] S. H. Sung, Marcinkiewicz-Zygmund type strong law of large numbers for pairwise i.i.d. random variables, J. Theor. Probab. 27 (2014), 96-106.

[24] R. L. Taylor, Stochastic convergence of weighted sums of random elements in linear spaces, Springer-Verlag, Berlin, 1978.

[25] R. L. Taylor, R. F. Patterson and A. Bozorgnia, A strong law of large numbers for arrays of rowwise negatively dependent random variables, Stochastic Anal. Appl. 20 (2002), 643-656.

[26] D. Wei and R. L. Taylor, Convergence of weighted sums of tight random elements, J. Multivariate Anal. 8 (1978), 282-294.

[27] Q. Y. Wu, Convergence properties of pairwise NQD random sequences, Acta Math. Sinica (Chin. Ser.) 45 (2002), 617-624, in Chinese.

[28] Q. Wu and Y. Jiang, The strong law of large numbers for pairwise NQD random variables, J. Syst. Sci. Complex. 24 (2011), 347-357. 
[29] Y. Wu and M. Guan, Mean convergence theorems and weak laws of large numbers for weighted sums of dependent random variables, J. Math. Anal. Appl. 377 (2011), 613-623.

[30] Y. Wu and D. Wang, Convergence properties for arrays of rowwise pairwise negatively quadrant dependent random variables, Appl. Math. 57 (2012), 463-476.

[31] G.-D. Xing, On the almost sure convergence rates for pairwise negative quadrant dependent random variables, Thai J. Math. 8 (2010), 171-184.

Y. Wu

College of Mathematics and Computer Science

Tongling University

244000 Tongling

China

and

Center for Financial Engineering and School of Mathematical Sciences

Soochow University

215006 Suzhou

China

E-mail: wyf wyf $@ 126 . c o m$

A. Rosalsky

Department of Statistics

University of Florida

Gainesville, FL 32611

USA

E-mail: rosalsky@stat.ufl.edu

Received: 26.2.2014.

Revised: 3.7.2014. 